\title{
Predictors of Percutaneous Endoscopic Gastrostomy Tube Placement after Stroke
}

\author{
Jian Li, Juan Zhang, Shujuan Li, Hongliang Guo, Wei Qin, Wen li Hu
}

\begin{abstract}
Aims: The goal of this study was to identify important prognostic variables affecting placement of a percutaneous endoscopic gastrostomy (PEG) tube after acute stroke. Methods: We retrospectively reviewed our patient database to identify acute ischemic stroke patients who placed PEG or nasogastric tube (NGT) tube, but were free of other confounding conditions affecting swallowing. A total of 340 patients were involved in our study. We assessed the influence of age, National Institutes of Health Stroke Scale (NIHSS) score, infarct volume, stroke subtype based on the TOAST criteria, swallowing disorders, bilateral lesions in cerebrum and length of stay (LOS) in a logistic regression analysis. Results: In univariate analysis, age ( $\mathrm{p}=0.048)$, NIHSS score (p<0.0001), lesion volume $(p<0.0001)$, LOS $(p<0.0001)$, stroke location $(p=0.045)$, and swallowing disorders $(p<0.0001)$ were found to be the primary predictors of placing PEG. The presence of lesions in bilateral cerebral was included in the final model based on clinical considerations. After multivariate adjustment, only NIHSS score (odds ratio [OR], 4.055; 95\% confidence interval [CI], 2.398-6.857; $\mathrm{p}=0.0001$ ), lesion volume (OR, 1.69; 95\%CI, 1.09-4.39; p=0.014), swallowing disorders (OR, 1.151; 95\% CI, 1.02-1.294; p=0.047), LOS (OR, 0.955; 95\% CI, 0.914-0.998; $\mathrm{p}=0.0415)$ and bilateral lesions (OR, 2.8; 95\% CI, 1.666-4.705; $\mathrm{p}=0.0001)$ remained significant. Conclusion: Our data shows that NIHSS score, lesion volume, swallowing disorders, LOS and bilateral lesions in cerebrum can predict the requiring of PEG tube insertion in patients after stroke.
\end{abstract}

RÉSUMÉ: Facteurs de prédiction de la mise en place d'un tube de gastrostomie par endoscopie percutanée après un accident vasculaire cérébral. Objectifs: Le but de cette étude était d'identifier les variables importantes du pronostic influençant la mise en place d'un tube de gastrostomie par endoscopie percutanée après un accident vasculaire cérébral aigu. Méthode : Nous avons revu rétrospectivement notre base de données pour identifier les patients atteints d'un AVC ischémique aigu, chez qui un tube nasogastrique ou un tube de gastrostomie par endoscopie percutanée avait été mis en place, mais qui ne présentaient pas d'autres problèmes touchant la déglutition. Notre étude porte sur 340 patients. Nous avons étudié au moyen d'une analyse de régression logistique l'influence de l'âge, le score au National Institutes of Health Stroke Scale (NIHSS), le volume de l'infarctus, le sous-type d'AVC selon les critères TOAST, les problèmes de déglutition, les lésions bilatérales au niveau des hémisphères cérébraux et le temps d'hospitalisation (TH). Résultats : À l'analyse univariée, l'âge $(\mathrm{p}=0,048)$, le score au NIHSS $(\mathrm{p}<0,0001)$, le volume de la lésion ( $\mathrm{p}<0$,0001), le TH $(\mathrm{p}<0,0001)$, l'endroit de l'AVC $(\mathrm{p}=0,045)$ et les troubles de la déglutition $(\mathrm{p}<0,0001)$ étaient les principaux facteurs de prédiction de la mise en place d'un tube de gastrostomie. La présence de lésions cérébrales bilatérales était incluse dans le modèle final pour des raisons cliniques. Après ajustement multivarié, seuls le score NIHSS (rapport de cotes [RC] 4,055; intervalle de confiance [IC] à 95\% de 2,398 à 6,857; p = 0,0001), le volume de la lésion (RC 1,69; IC à 95\% de 1,09 à 4,39; p = 0,014), les troubles de la déglutition (RC 1,151; IC à 95\% de 1,02 à 1,294; p = 0,047), le TH (RC 0,955; IC à $95 \%$ de 0,914 à 0,$998 ; p=0,0415)$ et les lésions bilatérales (RC 2,8; IC à 95\% de 1,666 à 4,705; p = 0,0001 demeuraient significatifs. Conclusion : Nos données démontrent que le score au NIHSS, le volume de la lésion, les troubles de la déglutition, le TH et la présence de lésions bilatérales dans les hémisphères cérébraux peuvent prédire la nécessité de mettre en place un tube de gastrostomie par endoscopie percutanée chez les patients atteints d'un AVC.

Can J Neurol Sci. 2014; 41: 24-28

Dysphagia are common after stroke and can cause chest infection and malnutrition. ${ }^{1}$ Therefore, patients with swallowing problems may need nutritional support. Enteral feeding offers one way of providing such support. ${ }^{2}$ It may be needed for a short time or for prolonged periods after acute stroke. Short-term feeding is usually given through a nasogastric tube (NGT). ${ }^{3}$ Percutaneous endoscopic gastrostomy (PEG), a minimally invasive technique that places a feeding tube into the stomach or jejunum, is used for longer periods. It is considered to be relatively simple and safe, while causing minimal discomfort. ${ }^{4}$ Each of these enteral feeding methods, however, has its own associated risks and benefits which must be considered. Nasogastric tube is easy to place, but it carries risks of tube displacement, aspiration pneumonia, and mechanical irritation of the nasopharynx, and can be uncomfortable for the patient as well. ${ }^{3,5}$ It is necessary to state that aspiration pneumonia is the most prevalent acute complication of dysphagia, affecting up to one-third of patients with acute ischemic stroke. Percutaneous endoscopic gastrostomy is a more desirable method for

From the Department of Neurology, Beijing Chaoyang Hospital, Capital Medical University, Beijing, China.

Received April 24, 2013. Final Revisions Submitted August 13, 2013. Correspondence to: Wen li Hu, Department of Neurology, Beijing Chaoyang Hospital, Capital Medical University, No. 8 South Gongti Road, Beijing 100020, China. Email: huwenli@sina.com. 
sustaining patient nutrition over longer periods, due to fewer tube failures ${ }^{6}$, lower risk complications, such as damage to the nose and larynx, chronic sinusitis, gastro-oesophageal reflux, and aspirative pneumonia. .,7 $^{6,}$

Identifying patients who fail to substantially improve swallowing function in early diagnosis would be beneficial for patients and their caregivers. Such patients can be placed on PEG earlier. The risk of aspiration pneumonia, length of stay (LOS), and cost of care can be reduced while nutrient absorption can be increased. However, PEG increases the risk of other complications, such as bowel perforation ${ }^{6,8,9}$. Up until now, there has been a lack of high quality evidence demonstrating the benefit of PEG in stroke patients. ${ }^{10,11}$

Some clinical observations have indicated that swallowing dysfunction predictors may include severe stroke as assessed by the National Institutes of Health Stroke Scale (NIHSS), ${ }^{3}$ different parts of brain regions (most notably the motor cortex, inferior frontal gyrus, insula, anterior insula, and periventricular white matter) ${ }^{12,13}$ age, ${ }^{14}$ and stroke lesion volume. ${ }^{15}$ However, the impact of these variables has not yet been systematically explored. ${ }^{16}$ The aim of our study was to analyze the effect of age, clinical severity (assessed by NIHSS score) and neuroanatomical correlates (including sites and lesion volume) in predicting PEG placement in patients with swallowing problems. In addition, we attempted to identify a new predictive factor for PEG placement.

\section{Material AND Methods}

Our study design was approved by Beijing Chaoyang Hospital's Institutional Review Board. In this retrospective and single-center study, patients were identified using Health Interventions codes-10 I63; I64; I65 and I66. Patients between January 2005 and January 2013 at Beijing Chaoyang Hospital were enrolled in the study. Inclusion criteria for patients included the presence of dysphagia, diagnostic accuracy cerebral infarction, as well as PEG or NGT placement during the same hospitalization. Each patient involved in our research underwent swallowing evaluation and a brain magnetic resonance imaging (MRI) scan. The stroke severity was assessed by the NIHSS when patients were admitted to the department of neurology in Beijing Chaoyang Hospital. We gathered information on patient demographics, including age, sex, stroke-associated vascular risk factors, history of transient ischemic attack or stroke, comorbidities (cardiac illnesses, diabetes, chronic hypertension, dyslipidemia), and common hospital complications (aspiration pneumonia, hospital mortality). Aspiration was defined as the entry of material into the airway below the levels of the true vocal cords. ${ }^{17}$ We also included the type of stroke (based on TOAST criteria) and the arterial distribution. Patients who had other confounding conditions that might have independently produced swallowing impairment, such as vocal cords dysfunction, prior tracheostomy, oropharyngeal malignancy, brain tumor, Parkinson's disease, advanced dementia, demyelinating diseases, inflammatory myopathies, myasthenia gravis, muscular dystrophies and cerebral hemorrhage, were excluded. Advanced dementia cognitive disorder eligibility criteria included age: 65 and older, dementia (any type, determined from the medical record), and a Global Deterioration Scale score of 7 (ascertained in an interview with a nurse caring for the resident). ${ }^{18}$ In order to maximize the reliability of the estimates and minimize the confounding factors, subjects from the NGT group were also excluded if they subsequently had PEG inserted.

The brain MRI scan was reviewed by a radiologist who was blinded to the clinical data. Lesion volume and location were analyzed on diffusion-weighted imaging (DWI) sequences of brain MRI images obtained between 24 and 72 hours after symptom onset. Lesion volume was obtained from the original medical record and verified by two independent neurologistobservers and two radiologists. We used the $\mathrm{ABC} / 2$ formula to evaluate the lesion volume. ${ }^{19}$ Observers were blinded to data measured lesions in three perpendicular axes. ${ }^{20}$ The slice with the largest lesion was first selected by eye. The longest lesion axis on this slice was measured with the ruler on an AGFA R4 Workstation with Impax Select software (v5205.0.0.1). A second line was drawn perpendicular to the first at the widest dimension. These two measurements were called the $\mathrm{x}(\mathrm{A})$ and $\mathrm{y}(\mathrm{B})$ axes. A third axis, the $\mathrm{z}(\mathrm{C})$ axis, was computed by multiplying the number of slices by slice thickness. ${ }^{19-21}$ The scan slice for MRI ranged from 6 to $7 \mathrm{~mm}$ for each subject was made by manually tracing the perimeter of the area on each MRI slice showing the infarct. ${ }^{19}$ This has been shown to be the most reproducible method of measuring lesion volume. ${ }^{19,20}$ All regions were scored aggregately in each individual patient.

The swallowing evaluation was done at bedside, including the repetitive saliva swallowing test (RSST) and modified water swallowing test (MWST). ${ }^{22,23}$ The tests were performed on the first day and the seventh day of hospitalization. These are excellent screening methods for assessing swallowing functions without using video fluoroscopy (VF), and their results correlate closely with those obtained using VF. ${ }^{22,24}$ The RSST and MWST were not performed in subjects with disturbed consciousness or unstable respiration, hypersecretion of sputum, or in those who were coughing intensely on their own saliva. In addition, the MWST was not performed in subjects who were unable to sit at

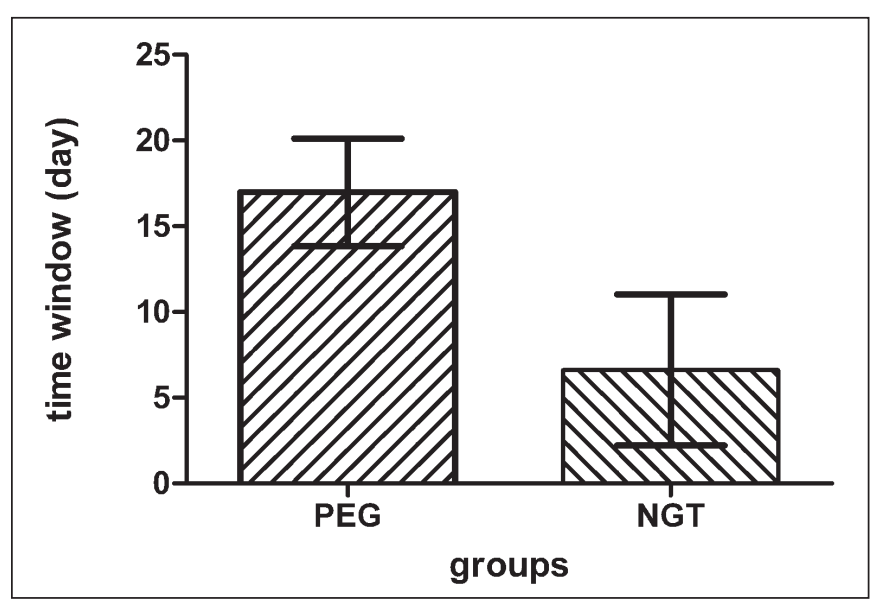

Figure: The mean time for PEG and NGT placement is shown in the Figure. The mean time for PEG placement was 16.98 days, and the mean time of NGT placement was 6.62 days after hospitalization. There was a significant difference between the two groups $(P<0.0001)$. 
$90^{\circ}$ or who had fever or pneumonia. In this study, patients in the acute phase of stroke were classified as having normal swallowing function if RSST within 30 seconds was $\geq 3$ and the MWST was $\geq 4$; swallowing impairment was defined as a RSST within 30 seconds of $\leq 2$ or a MWST result of $\leq 3$. The exact time for PEG and NGT placement were decided by two gastroenterologists who were blind to the diagnosis and treatment of the patients.

\section{Statistical Analysis}

Variables were analyzed by univariate and then multivariate logistic regression using SPSS software 17.0. For the purpose of statistical analysis, two continuous variables - age and infarct volume - were dichotomized into two groups each (age,$<$ and $\geq 75$ years; infarct volume,$<$ and $\geq 100 \mathrm{~mm}$ ) to improve clinical interpretability. All remaining variables were entered in the analysis as binary variables (present/absent). Multivariate modeling included any variable found to have a $\mathrm{P}$ value $<0.05$ in univariate analysis; the lesions in bilateral cerebrum were included in the final model based on clinical considerations.

\section{RESUlts}

In our study there were 350 potential patients, 10 of them had NGT before PEG placement. These ten subjects were excluded from the study. The final analysis included 340 patients. The times of PEG and NGT placement are shown in the Figure. The mean time for PEG placement was 16.98 days, and the mean time of NGT placement was 6.62 days after hospitalization. There was a significant difference between the two groups $(\mathrm{P}<$ $0.0001)$.

Table: The baseline characteristics of patients and risk factors associated with PEG and NGT placement

\begin{tabular}{|c|c|c|c|c|}
\hline Variable & Study cohort & PEG（57） & NGT（283） & P value* \\
\hline age, years, mean $\pm \mathrm{SD}$ & $69.00 \pm 17.03$ & $73.2 \pm 8.9$ & $64.7 \pm 8.1$ & $0.048^{*}$ \\
\hline Female $(\%)$ & $178(52.25 \%)$ & 35 & 143 & 0.069 \\
\hline Male (\%) & $162(47.65 \%)$ & 22 & 140 & 0.076 \\
\hline NIHSS score, mean \pm SD & $13.32 \pm 9.4$ & $19.01 \pm 6.1$ & $8.03 \pm 8.21$ & $<0.0001^{*}$ \\
\hline Stroke volume $\left(\mathrm{cm}^{3}\right)$, mean $\pm \mathrm{SD}$ & $48.02 \pm 12.3$ & $76.2 \pm 13.2$ & $34.8 \pm 11.2$ & $<0.0001^{*}$ \\
\hline Length of stay, mean $\pm \mathrm{SD}$ & $18.25 \pm 9.36$ & $28.1 \pm 10.1$ & $12.9 \pm 5.9$ & $<0.0001^{*}$ \\
\hline Stroke subtype based on TOAST criteria, n (\%) & & & & 0.006 \\
\hline Large vessel atherosclerosis & $93(27.4 \%)$ & $11(19.3 \%)$ & $82(29.0 \%)$ & \\
\hline Cardio embolism & $114(33.5 \%)$ & $41(71.9 \%)$ & $73(25.8 \%)$ & \\
\hline Small vessel occlusion & $54(15.9 \%)$ & $3(0.5 \%)$ & $51(18.0 \%)$ & \\
\hline Stroke of other determined etiology & $28(8.2 \%)$ & $2(0.4 \%)$ & $26(9.2 \%)$ & \\
\hline Stroke of undetermined etiology & $48(14.1 \%)$ & 0 & $48(17.0 \%)$ & \\
\hline Stroke location by arterial distribution, n (\%) & & & & $0.045^{*}$ \\
\hline Middle cerebral artery & $221(65.0 \%)$ & $42(73.7 \%)$ & $179(63.3 \%)$ & \\
\hline Posterior cerebral artery & $11(3.2 \%)$ & $2(3.5 \%)$ & $9(3.2 \%)$ & \\
\hline Anterior cerebral artery & $24(7.1 \%)$ & $3(5.3 \%)$ & $21(7.4 \%)$ & \\
\hline Vertebrobasilar artery & $39(11.5)$ & $7(12.3 \%)$ & $32(11.3 \%)$ & \\
\hline Watershed infarction & $16(4.7 \%)$ & $1(1.8 \%)$ & $15(5.3 \%)$ & \\
\hline Multifocal circulation & $39(11.5 \%)$ & $2(3.5 \%)$ & $27(9.5 \%)$ & \\
\hline Hospital complications, & & & & 0.054 \\
\hline Hospital mortality & 10 & 1 & 9 & \\
\hline Pneumonia & 103 & 18 & 85 & 0.64 \\
\hline Diabetes & 175 & 29 & 146 & 0.67 \\
\hline Chronic hypertension & 172 & 33 & 139 & 0.72 \\
\hline Dyslipidemia & 211 & 37 & 174 & 0.77 \\
\hline
\end{tabular}

* Statistical significance provided for the PEG group versus NGT group. 
The baseline characteristics of patients and risk factors associated with PEG and NGT placement are shown in the Table. Males comprised slightly less than half of the total population, but both groups had similar sex proportions and hospital complications. The mean \pm SD NIHSS score was 13.32 \pm 9.4 , indicating a moderately severe stroke burden. The incidence of middle cerebral artery infarction was $33.5 \%$ in the included patients. In addition, there was no significant difference between the embolic disease group and the large vessel atherosclerosis group.

The most common hospital complications were hypoproteinemia and pneumonia. More than half of the patients suffered from at least one disease, such as diabetes, chronic hypertension, or dyslipidemia. Some patients even suffered from more than two of these diseases. Little difference was displayed between the two groups $(\mathrm{P}=0.048)$.

After univariate analysis, age, NIHSS score, infarct volume, LOS, stroke location based on arterial distribution and oropharyngeal dysphagia $(\mathrm{P}<0.0001)$ were found to be significantly associated with PEG placement. In a previous anatomical analysis, bilateral infarcts were significantly associated with oropharyngeal dysphagia and aspiration. ${ }^{3}$ Based on this consideration, we specifically assessed bilateral cerebral lesions (cerebral hemisphere and brain stem), whether the infarct was new or old. In the final multivariate model, we analyzed the influence of the aforementioned significant variables on our outcome of interest. The consequences of our study suggest that NIHSS score $(\mathrm{P}<0.0001)$, lesion volume $(\mathrm{P}=0.014)$, deglutition disorders $(\mathrm{P}=0.047), \mathrm{LOH}(\mathrm{P}=0.0415)$, and lesions in bilateral cerebrum $(\mathrm{P}<0.0001)$ are significantly associated with $\mathrm{PEG}$ placement. Age $(\mathrm{P}=0.55)$, cardio embolism $(\mathrm{P}=0.88)$ and middle cerebral artery $(\mathrm{P}=0.74)$, which appeared to associate with $\mathrm{PEG}$, didn't reach statistical significance.

\section{DiscusSION}

To our knowledge, this was the largest reported multivariate analysis on prognostic factors that affected PEG placement in acute ischemic stroke patients in China. One of the most important observations of our study was the effect of lesions in bilateral cerebrum in oropharyngeal dysphagia patients.

The main results of our study showed that after controlling and correcting for possible confounding effects, the NIHSS score, lesion volume, infarcts in bilateral cerebrum and $\mathrm{LOH}$ were significantly associated with PEG tube insertion. The presence of infarcts in bilateral cerebrum (hemispheres or brain stem) in stroke patients was 2.8 times higher in those with PEG tube insertion compared to those with NGT insertion. The results indicated that oropharyngeal swallowing functions were under bilateral brain control. Furthermore, this result confirmed the crucial role of the uninjured hemisphere or brain stem in mediating swallowing function by way of compensatory reorganization of its swallowing cortex. ${ }^{24,25}$

Ischemic stroke volume has been shown to be an important predictor of stroke recovery. A recent study found stroke volume assessed by DWI sequences was a significant variable in predicting outcome. ${ }^{26} \mathrm{We}$ also investigated the influence of lesion volume on the decision for or against PEG placement. Our results indicated that lesion volume had a significant impact on the decision for PEG insertion.
In hemispheric stroke patients with swallowing irregularities, Daniels et $a l^{27}$ found no significant association between severity of dysphagia and size of the stroke lesion using semiquantitative analysis with head computed tomogram (CT) scans, while Kumar et $a l^{14}$ detected that there was a relationship between dysphagia and hemispheric lesion volume. However, both of these studies calculated the volume of hemispheric lesion only. ${ }^{14,28,29}$ In our study, we measured volume of lesions in both cerebral hemispheres and brainstems. In addition, our study employed more quantitative methods and measured ischemic lesion volumes on DWI sequences of brain MRI images, which was a more reliable method of detecting cerebral ischemia. We further oriented the affect of lesion volume in order to obtain more applicable effects in multivariate analysis. After controlling for potential confounding effects, lesion volume showed a significant correlation with PEG placement in stroke patients. Previous studies of global measures for functional recovery have found that NIHSS score is an important predictor of recovery following stroke after adjusting for other variables. ${ }^{3,30}$ Our results are consistent with previous study results.

Our study had some limitations. First, our results indicated that the lesion volume had significant impact on the swallowing function. However, the results did not take into account how different infarct sites or how involvement of multiple such sites affected the chance of PEG placement. The use of more sophisticated and newer imaging techniques, such as voxelbased symptom lesion mapping and diffusion tractography, might help answer these questions in the future. Second, although our study suggests that the lesion volume has significant impact on swallowing function, our sample size was not sufficiently large enough to involve all these variables in our analysis. In addition, we did not collect the data about patients who had swallowing impairment without PEG placement. There were two reasons for this. First, this was a retrospective study, and it was difficult to collect patients who had swallowing impairment without PEG placement. Second, the goal of this study was to identify important prognostic variables affecting placement of a PEG tube after acute stroke. Thus, patients who had swallowing impairment but did not receive a PEG tube were not included in our study. We had attempted to minimize the impact of these influences by using a systematic method for identifying consecutive ischemic stroke patients with severe dysphagia using ICD-10. Nevertheless, we were unable to capture all such patients systematically by using our approach. This might have inadvertently introduced a selection bias. Finally, in order to illustrate OR in clinical data, our study dichotomized ischemic lesion volume. The categories chosen $\left(<100 \mathrm{~mm}^{3}\right.$ and $\left.\geq 100 \mathrm{~mm}^{3}\right)$ were based on the distribution of infarct volumes to ensure adequate numbers of patients in each group. Dichotomizing the continuous variable might also have compromised our statistical power and resulted in a more backward-looking estimate of effect on swallowing disorders. An appropriate solution to this conundrum might be to classify patients into more subcategories (eg, $15 \mathrm{~mm}^{3}$ categories). However, we were restricted by our sample capacity. Despite these limitations, our analysis improves the understanding of the influence of clinical and radiological variables in predicting PEG placement in the early stage following stroke. Clinicians should 
not wait for pneumonia to occur to make a decision regarding PEG placement.

In clinical practice, there is usually a delay of PEG tube insertion after ischemic stroke, which will prolong the length of stay. Identifying the variables discussed in this paper is an important step because building predictive models can capture individual information and assist physicians in making an educated decision. It is worthwhile for medical doctors to select patients who are suitable for PEG tube insertion at an early stage. We hope that our finding will facilitate larger, prospective studies that are better powered to detect the respective influences of these predictive factors.

\section{ACKNowledgments}

This work was funded by National Natural Science Foundation of China (NSFC), China (No.81271309), and by Beijing natural science foundation, China (7132070).

\section{REFERENCES}

1. Haywood S. PEG feeding tube placement and aftercare. Nurs Times. 2012;108:20-2.

2. Potack JZ, Chokhavatia S. Complications of and controversies associated with percutaneous endoscopic gastrostomy: report of a case and literature review. Medscape J Med. 2008;10:142.

3. Alshekhlee A, Ranawat N, Syed TU, Conway D, Ahmad SA, Zaidat OO. National Institutes of Health Stroke Scale assists in predicting the need for percutaneous endoscopic gastrostomy tube placement in acute ischemic stroke. J Stroke Cerebrovasc Dis. 2010;19:347-52.

4. Dennis MS, Lewis SC, Warlow C, et al. Effect of timing and method of enteral tube feeding for dysphagic stroke patients (FOOD): A multicentre randomised controlled trial. Lancet. 2005;365:764-72.

5. Panos MZ, Reilly H, Moran A, et al. Percutaneous endoscopic gastrostomy in a general hospital: prospective evaluation of indications, outcome, and randomised comparison of two tube designs. Gut. 1994;35:1551-6.

6. Gomes Jr CAR, Lustosa SAS, Matos D, Andriolo RB, Waisberg DR, Waisberg J. Percutaneous endoscopic gastrostomy versus nasogastric tube feeding for adults with swallowing disturbances. Cochrane Database of Systematic Reviews 2012, Issue 3.

7. Dwolatzky T, Berezovski S, Friedmann R, et al. A prospective comparison of the use of nasogastric and percutaneous endoscopic gastrostomy tubes for long-term enteral feeding in older people. Clin Nutr. 2001;20:535-40.

8. John B, Jesper L, Lena M, et al. Complications after percutaneous endoscopic gastrostomy in a prospective study. Scand J Gastroentero. 2012;47:737-42.

9. Somi MH, Maghbouli L, Antikchi M. Acceptability and outcomes of percutaneous endoscopic gastrostomy (PEG) tube placement and patient quality of life. Turk J Gastroenterol. 2011;22:128-33.

10. Dwolatzky T, Berezovski S, Friedmann R, et al. A prospective comparison of the use of nasogastric and percutaneous endoscopic gastrostomy tubes for long-term enteral feeding in older people. Clin Nutr. 2001;20:535-40.

11. Norton B, Homer-Ward M, Donnelly MT, Long RG, Holmes GK. A randomised prospective comparison of percutaneous endoscopic gastrostomy and nasogastric tube feeding after acute dysphagic stroke. BMJ. 1996;312:13-6.
12. Hilker R, Poetter C, Findeisen N, et al. Nosocomial pneumonia after acute stroke: Implications for neurological intensive care medicine. Stroke. 2003;34:975-81.

13. El Solh A, Okada M, Bhat A, Pietrantoni C. Swallowing disorders post orotracheal intubation in the elderly. Intensive Care Med. 2003;29:1451-5

14. Kumar S, Langmore S, Goddeau RP Jr, et al. Predictors of percutaneous endoscopic gastrostomy tube placement in patients with severe dysphagia from an acute-subacute hemispheric infarction. J Stroke Cerebrovasc Dis. 2012;21:114-20.

15. Bates B, Choi JY, Duncan PW, et al. Veterans Affairs/Department of Defense clinical practice guideline for the management of adult stroke rehabilitation care: Executive summary. Stroke. 2005;36: 2049-56.

16. Martin RE, Goodyear BG, Gati JS, Menon RS. Cerebral cortical representation of automatic and volitional swallowing in humans. J Neurophysiol. 2001;85:938-50.

17. Ertekin C, Aydogdu I. Neurophysiology of swallowing. Clin Neurophysiol. 2003;114:2226-44.

18. Reisberg B, Ferris SH, Georgotas A, et al. The global deterioration scale for assessment of primary degenerative dementia. Am J Psychiatry. 1982;139:1136-9.

19. Sims JR, Gharai LR, Schaefer PW, et al. $\mathrm{ABC} / 2$ for rapid clinical estimate of infarct, perfusion, and mismatch volumes. Neurology. 2009;72:2104-10.

20. Pedraza S, Puig J, Blasco G. Reliability of the $\mathrm{ABC} / 2$ method in determining acute infarct volume. J Neuroimaging. 2012;22: $155-9$.

21. Hamdy S, Aziz Q, Rothwell JC, et al. Explaining oropharyngeal dysphagia after unilateral hemispheric stroke Lancet. 1997;350: 686-92.

22. Osawa A, Maeshima S, Matsuda H, Tanahashi N. Functional lesions in dysphagia due to acute stroke: discordance between abnormal findings of bedside swallowing assessment and aspiration on videofluorography. Neuroradiology. 2013;55:413-21.

23. Baba Y, Teramoto S, Hasegawa H, Machida A, Akishita M, Toba K. Characteristics and limitation of portable bedside swallowing test in elderly with dementia: comparison between the repetitive saliva swallowing test and the simple swallowing provocation test. Nihon Ronen Igakkai Zasshi. 2005;42:323-7.

24. Osawa A, Maeshima S, Tanahashi N. Water-swallowing test: screening for aspiration in stroke patients. Cerebrovasc Dis. 2013;35:276-81.

25. Hamdy S, Aziz Q, Rothwell JC, et al. Recovery of swallowing after dysphagic stroke relates to functional reorganization in the intact motor cortex. Gastroenterology. 1998;115:1104-12.

26. Johnston KC, Wagner DP, Wang XQ, et al. Validation of an acute ischemic stroke model: Does diffusion-weighted imaging lesion volume offer a clinically significant improvement in prediction of outcome? Stroke. 2007;38:1820-5.

27. Daniels SK, Foundas AL. Lesion localization in acute stroke patients with risk of aspiration. Neuroimaging. 1999;9:91-8.

28. Turhan N, Atalay A, Muderrisoglu H. Predictors of functional outcome in first-ever ischemic stroke: A special interest to ischemic subtypes, comorbidity and age. NeuroRehabilitation. 2006;24:321-6.

29. Thijs VN, Lansberg MG, Beaulieu C, et al. Is early ischemic lesion volume on diffusion-weighted imaging an independent predictor of stroke outcome? A multivariable analysis. Stroke. 2000;31: 2597-602.

30. Lindsell CJ, Alwell K, Moomaw CJ, et al. Validity of a retro spective National Institutes of Health Stroke Scale scoring methodology in patients with severe stroke. J Stroke Cerebrovasc Dis. 2005; $14: 281-3$. 